\title{
Dynamic response of a plate laying on elastic base during the impact of a conical indenter
}

\author{
Alexey Beskopylny ${ }^{1, *}$, Andrey Veremeenko ${ }^{1}$, Elena Kadomtseva ${ }^{1}$, and Alexander Shilov ${ }^{1}$ \\ ${ }^{1}$ Don State Technical University, pl. Gagarin, 1, Rostov-on-Don, 344010, Russia
}

\begin{abstract}
The article presents an analytical and numerical study of a dynamic conical indentation during impact process. The circular plate laying on the elastic base is subjected to impact load by the conical indenter. Local deformations in imprint zone are considered as elasticplastic and general plate deformations are only elastic. According to Timoshenko method, the general plate displacement is considered as the sum of the elastic-plastic displacement of the indenter and the elastic displacement of the plate at the impact point. The solution is constructed with Fourier transforms and the Green function of the dynamic process is obtained. The numerical study of impact indentation is received by FEM method. The dependences of displacements, velocities, and accelerations of the plate on time is obtained during impact. The experimental data obtained with the special device is compared with the results of the numerical analysis. The method of an assessment of the mechanical characteristics of steel is proposed on the basis of the research. Analysis of the dynamic response and non-stationary effects on structural elements is carried out on the base of the calculated impact process.
\end{abstract}

\section{Introduction}

The problem of the dynamic response of a plate laying on an elastic base is widely applied in the construction field. The most dangerous are dynamic or impact loads that can cause critical damage to a structure. Such models are widely used in practice in construction, road and highway design, mechanical engineering, the theory of composite structures, in the production of body armor and many other areas.

Depending on an application area, it is advisable to distinguish three ranges of impact speed. First is low-velocity impact till $10 \mathrm{~m} / \mathrm{s}$. It is considered that in this case, wave processes and inertial forces in the imprint area do not have a significant influence on the reaction of the material and they can be neglected. The second is a high-velocity impact from $10 \mathrm{~m} / \mathrm{s}$ to $100 \mathrm{~m} / \mathrm{c}$. In this case, the inertial resistance of the material increases, and the mechanical characteristics of the material change significantly. And the third is ultra-high speed. The impact resistance of the material increases even more and can reach the limit of elasticity.

In this paper, we consider low-velocity impact as the most common in construction practice and can be used for non-destructive testing of structures. The method of non-

\footnotetext{
${ }^{*}$ Corresponding author: besk-an@ya.ru
} 
destructive test is considered in [1-3] on the basis of conical indentation and the number of mechanical characteristics is determined with the special device.

The dynamic response of a plate laying on a soil medium subjected to a moving load is analytically obtained under conditions of plane strain in $[4,5]$. The plate is assumed to be isotropic, linear elastic and obeying Kirchhoff's theory of bending.

The simple expression for the dynamic response of stiffened rectangular plates repeatedly impacted by a rigid knife-edged strike was proposed in [6]. The method was based on the hypothesis of the rigid-perfectly plastic body to examine the influence of the mechanical properties on theoretical prediction of the impact parameters.

The dynamic response of polycarbonate plates subjected to the spherical indenter impact in different velocities was investigated in [7]. The numerical investigation of impact response of a polycarbonate plate is conducted by using LS-DYNA. Polycarbonate material behaves in an elastoplastic manner. The velocities were in a range of $50-120 \mathrm{~m} / \mathrm{s}$.

The analytical and numerical investigation of the stress-strain state of the corrugated plate on an elastic foundation by the Bubnov-Galerkin method is obtained in $[8,9,10]$. The displacements are expressed in the form of an expansion in a series with unknown coefficients by special polynomials, which are a combination of Legendre polynomials. Dynamic behavior of structural elements allows estimating risks of sudden structural failure [11,12].

All cases show that the problem of the impact of a hard body on the plate under elastoplastic deformation is a relevant task and the assessment of the parameters of statestrain state of the plate is a necessary part of the dynamic research.

\section{Materials and methods}

Let's consider a circular plate with the radius $L$ and thickness $H$ lying on the elastic base of Winkler with a stiffness coefficient $k_{0}$. The mechanical characteristics of a plate resume to be elastic perfectly plastic with the yield strength $\sigma_{y}$. A conical body with mass $m$ moving at a velocity $v_{0}$ and hit in the center of the plate with angle $90^{\circ}$. We consider the cylindrical coordinate system $\operatorname{0r} \varphi \xi$, the origin of which is coincides at the point of initial contact. The local deformations in the contact zone are elastoplastic and general deformations of the plate are considered elastic. It's necessary to determine main characteristics of impact: the stress-strain state and kinematic parameters - the displacement $S(t)$, the velocities $V(t)$, the accelerations $W(t)$ during impact.

The differential equation of motion of a plate subjected to contact force $P(t)$

$$
D \Delta^{2} w+k_{0} w+\rho_{1} h \frac{\partial^{2} w}{\partial t^{2}}=P(t) \delta(r), \quad \Delta=\frac{\partial^{2}}{\partial r^{2}}+\frac{1}{r} \frac{\partial}{\partial r}
$$

where $w(r, \varphi)$ the displacement of the plate, $P(t)$ - contact force, $D$ - the plate cylindrical stiffness.

The boundary conditions for a plate with a free edge have the form

$$
\Delta w+\left(v_{1}-1\right) \frac{1}{r} \frac{\partial w}{\partial r}=0, \frac{\partial \Delta w}{\partial r}=0 \text { when } r=L
$$

The motion $z(t)$ of the impactor is described by the equation

$$
m \ddot{z}=-P(t), z(0)=0, \dot{z}(0)=\dot{v}_{0}
$$


The dependence of the contact force $P(t)$ is taken from the static solution of conical indentation $P(\alpha)$ [3]. In view of the linearity of the problem, it suffices to consider the action of a unit pulse $Y=1$. The movement in this case will be a function of influence $f(r, t)$, and the displacement at the origin of coordinates under the action of the concentrated impact force is determined by the equation

$$
w(r, t)=\int_{0}^{t} P(s) f(r, t-s) d s
$$

This solution is written in explicit form. As a result, the Green's function has the following form

$$
\begin{gathered}
f(\tau)=\frac{8 \sin (\omega \tau)}{\pi \omega}+4 \sum \frac{A_{n}}{\lambda_{n}} \sin \left(\lambda_{n} \tau\right) \\
\omega=L^{2}\left(\frac{k_{0}}{D}\right)^{1 / 2}, \lambda_{n}=\left(u_{n}^{4}+\omega^{2}\right)^{1 / 4}, u_{0}=0, \lambda_{0}=\omega, u^{4}=-\left(s^{2}+\omega^{2}\right) \\
T_{2}(u)=-\frac{4}{\pi u}+J_{0}(u) N_{1}(u)+I_{1}(u) N_{0}(u)+\frac{2}{\pi}\left[J_{1}(u) K_{0}(u)-J_{0}(u) K_{0}(u)\right] \\
-2\left(1-v_{1}\right)\left[I_{1}(u) N_{1}(u)-\frac{2}{\pi u} J_{1}(u) K_{1}(u)\right] \\
A_{n}=-\frac{u_{n} T_{2}\left(u_{n}\right)}{\left[U_{2}(u)\right]^{\prime}\left(u_{n}\right)}, U_{2}(u)=J_{0}(u) I_{1}(u)+J_{1}(u) I_{0}(u)-2\left(1-v_{1}\right) \frac{J_{1}(u) I_{1}(u)}{u}
\end{gathered}
$$

$I_{i}(u), K_{i}(u), i=0,1$ - modified Bessel and Hankel functions of the $i$-th order; $J_{i}(u), N_{i}(u), i=0,1$ - Bessel and Neumann functions of the $i$-th order.

Further investigation was carried out numerically with the FEM method using ANSYS soft. The scheme (figure 1) presents impact testing. The striker 2 hits over the indenter 3 subjected to the spring 1 force and sensor registers a signal of velocity $V(t)$ of the plate 4 . The displacements $S(t)$ and accelerations $W(t)$ are obtained by integrating and differentiating. The FEM model of a task of the impact of a conical indenter on a plate in an axisymmetric state was produced. Considering that during the impact there is a significant plastic deformation in the contact zone, the plate was modeled by rectangular 8 -node elements with the orthotropic material properties. There was more frequent finite element grid meshing in the contact zone of the plate with the impactor. written

The general equation of motion for the discrete mechanical system (figure 1) can be

$$
\mathbf{M} \ddot{\mathbf{u}}_{n+1}+\mathbf{C} \dot{\mathbf{u}}_{n+1}+\mathbf{K} \mathbf{u}_{n+1}=\mathbf{F}_{n+1}^{a}
$$

$\mathbf{M}$ - mass matrix, $\mathbf{C}$ - damping matrix, $\mathbf{K}$ - stiffness matrix, $\mathbf{F}^{a}$ - applied load vector, $\ddot{\mathbf{u}}_{n+1}$ - the nodal acceleration vector at time $t_{n+1}, \dot{\mathbf{u}}_{n+1}-$ the nodal velocity vector, $\mathbf{u}_{n+1}-$ the nodal displacement vector. The integration algorithm for (5) by Newmark method was used. 

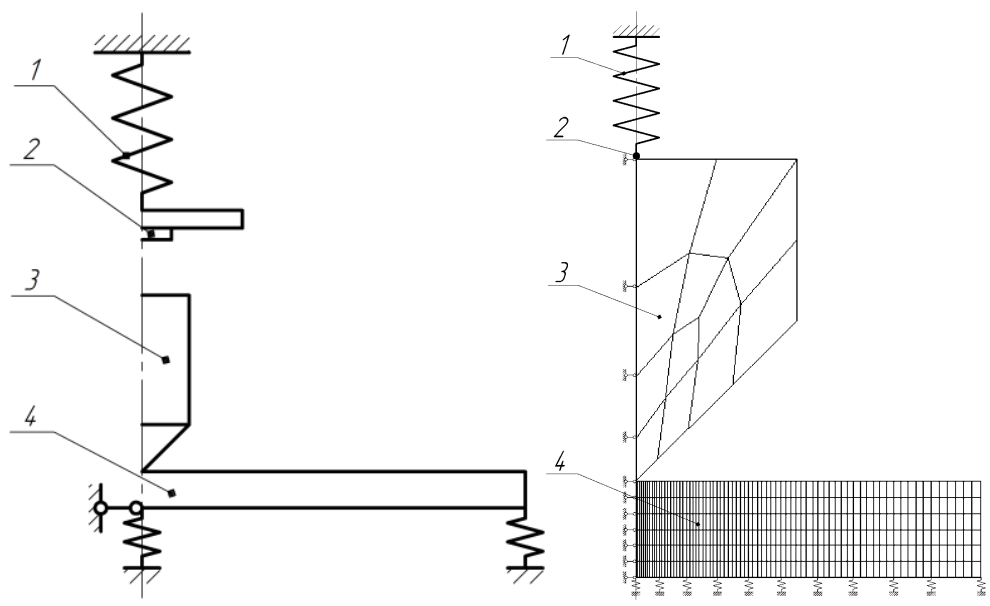

Fig. 1. The scheme of impact (left) and FEM model (right).

\section{Results}

The steel plate with diameter $2 L=94 \mathrm{~mm}$ and thickness $H=3 \mathrm{~mm}$ on the elastic base (rubber) $k=1282 \mathrm{~N} / \mathrm{m}$. The elastic base was modeling as a layer with elasticity modulus $E_{\mathrm{b}}=3.0 \mathrm{E}+6 \mathrm{MPa}$. The yield strength of steel plate $\sigma_{y}=330 \mathrm{MPa}$. The striker with mass $m_{\mathrm{s}}=0.090 \mathrm{~kg}$, hits on the indenter with mass $m_{\mathrm{i}}=0.080 \mathrm{~kg}$. The energy of striker $E=0.16 \mathrm{~J}$.

The results of numerical analysis and experimental data are shown in figure 2 . The dependences of displacement $S(t)$, velocity $V(t)$ and acceleration $W(t)$ are presented in figure 2 where the experimental data are compared with the ANSYS results. It's seen that in the active phase of the impact all dependencies almost coincide. The discrepancy appears during the rebound of the indenter that is well seen at the second dependence $V(t)$ (figure 2) when the speed curve is reduced to zero.

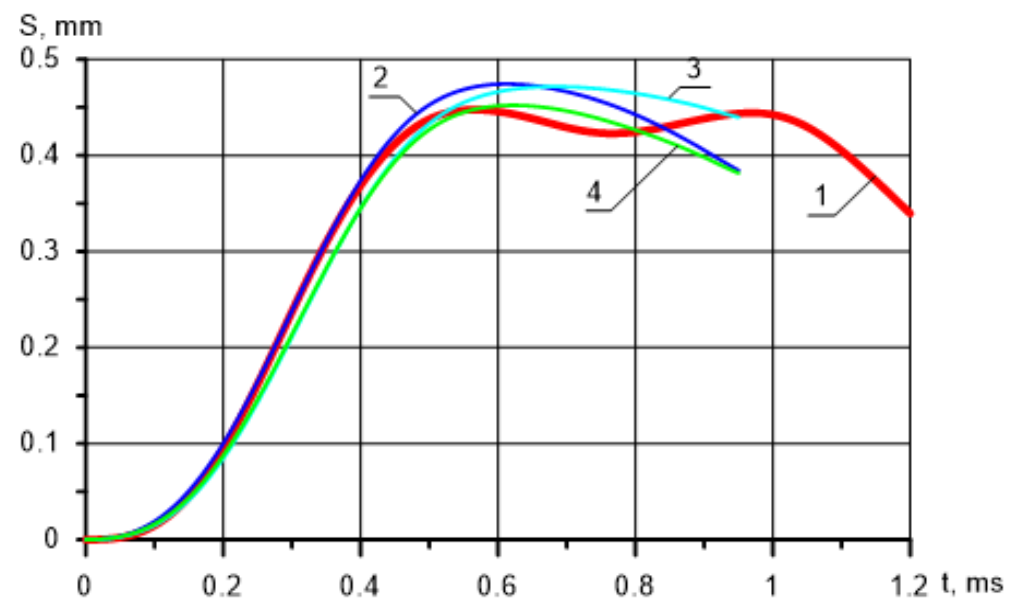

a) 


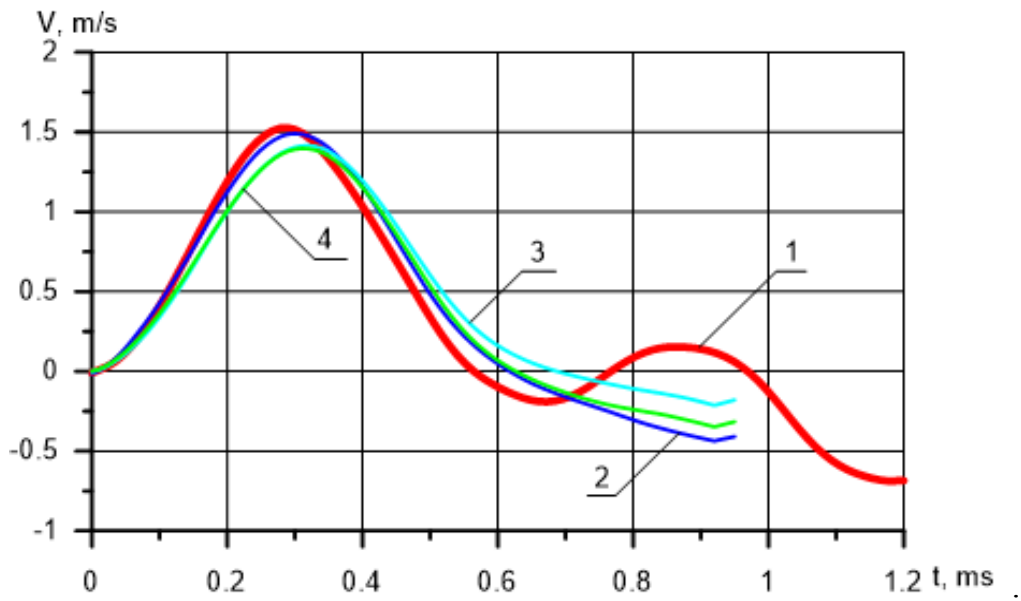

b)

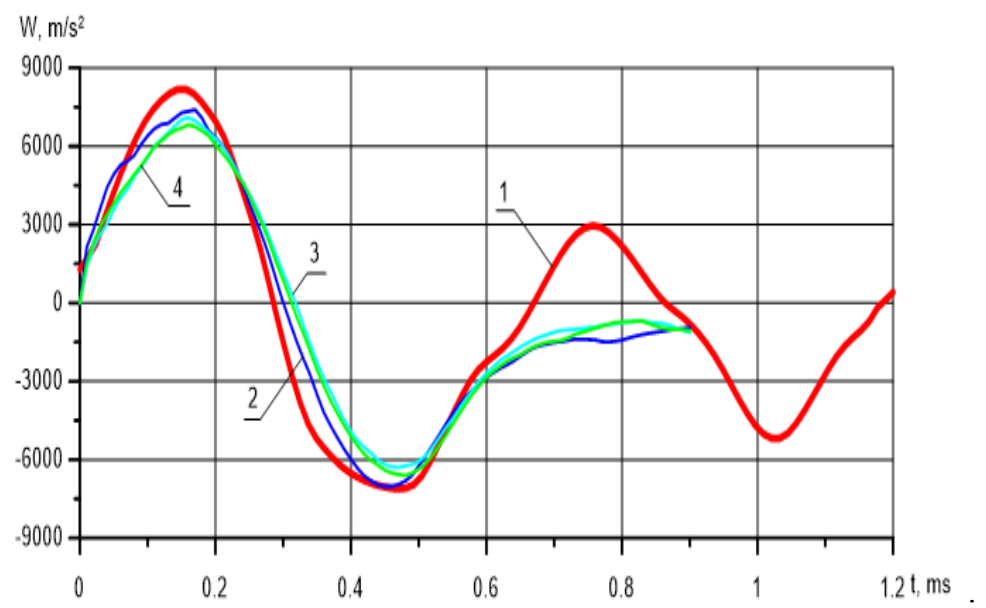

c)

Fig. 2. The dependences a) of displacement $S(t), b)$ velocity $V(t)$ and $c)$ acceleration $W(t)$ during impact: 1- numerical analysis, 2 - 1 experiment, 3 - 2 experiment, 4 - 3 experiment.

The discrepancy between the graphs in the passive part of the experiment, when the indenter is rebound, can be explained by the fact that the rubber base has a damping property that was not considered in the numerical analysis. Nevertheless, we see a good agreement of the results in the first half-wave of plate oscillations. The experiment was carried out on a specially manufactured unit [3].

\section{Discussion}

The plate response for various elastic coefficients of the base is considered during impact conical indentation. Figure 3 shows the dependences of displacement $S(t)$, velocity $V(t)$ and acceleration $W(t)$ for three kinds of elastic foundation $E_{1}=3.0 \mathrm{E}+5 \mathrm{MPa}, E_{2}=3.0 \mathrm{E}+6 \mathrm{MPa}$, $E_{3}=3.0 \mathrm{E}+7 \mathrm{MPa}$. 


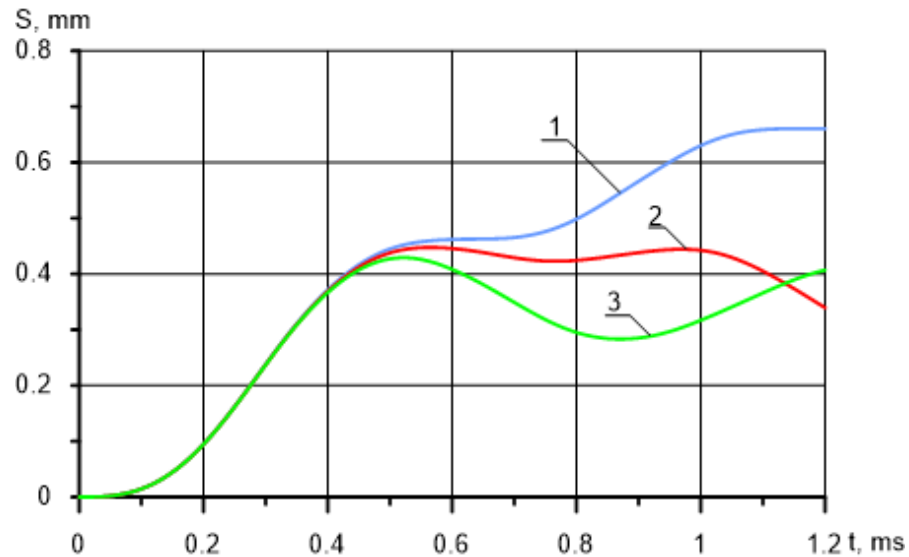

a)

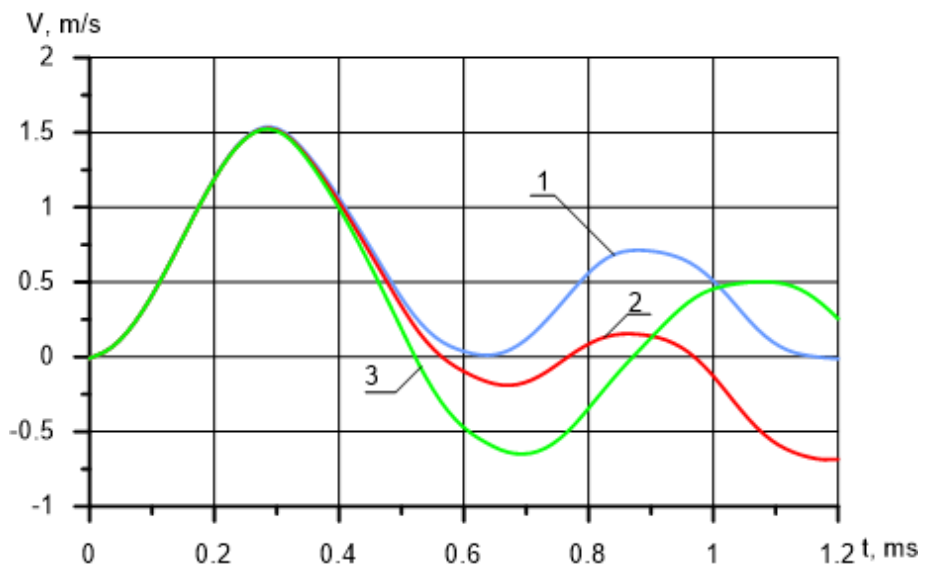

b)

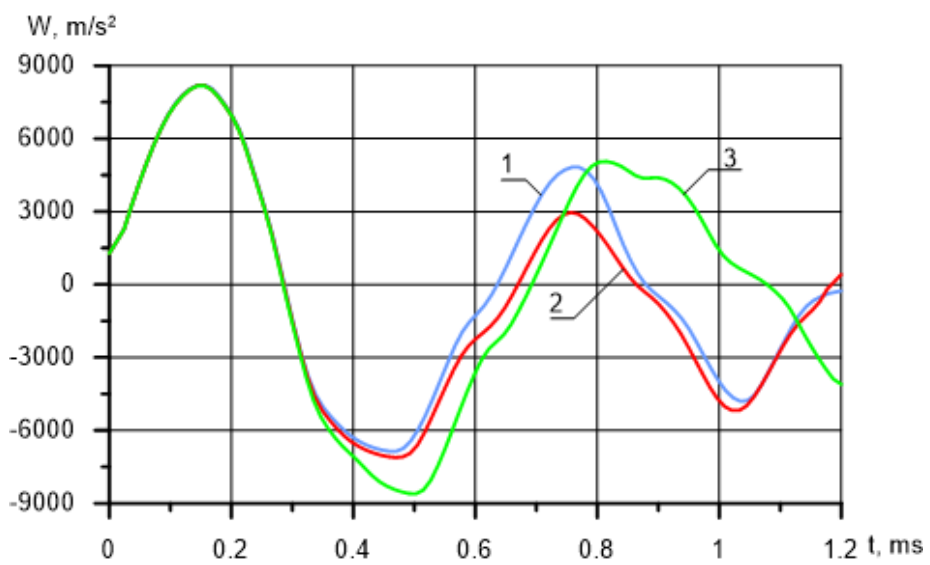

c)

Fig. 3. a) The displacement $S(t)$, b) velocity $V(t)$ and c) accelerations $W(t)$ during the impact for different base stiffness: $1-E_{1}=3.0 \mathrm{E}+5 \mathrm{MPa}, 2-E_{2}=3.0 \mathrm{E}+6 \mathrm{MPa}, 3-E_{3}=3.0 \mathrm{E}+7 \mathrm{MPa}$

The dynamic response of the plate to the impact of the indenter is a complex function because in the scheme adopted by us, the indenter is initially resting on the plate and the striker hits it with a given energy. In this case, the dynamics of the plate is determined not 
only by the properties and geometry of the plate and the impact mechanism is a two-mass system.

\section{Conclusions}

The method for dynamic plate response during impact conical indentation has been developed. The numerical analysis was compared with experimental data and it's shown that given data are in good agreement in the active phase of indentation.

The numerical analysis of the dynamic response of a circular plate on an elastic base was produced for various elastic foundation modulus. The proposed method $3 \mathrm{~d}$ is tested on non-destructive testing of structural elements [3], welded joints [13], machine parts [14] and others.

The work was financially supported by Russian Foundation for Base Research (project 18-01-00715-a).

\section{References}

1. A.N. Beskopylny, A.A. Veremeenko, B.M. Yazyev, MATEC Web of Conferences 106, 04004 (2017)

2. A.N. Beskopylny, A.A. Lyapin, V.I. Andreev, MATEC Web of Conferences 117, 00018 (2017)

3. A. Beskopylny, A. Veremeenko, E. Kadomtseva, N. Beskopylnaia, MATEC Web of Conferences 129, 02046 (2017)

4. Yuyan Chen, Niki D. Beskou, Jiang Qian, Soil Dynamics and Earthquake Engineering 107, 292-302 (2018)

5. Niki D. Beskou, Yuyan Chen, Jiang Qian, Transportation Geotechnics 14, 98-106 (2018)

6. Ling Zhu, Shiyun Shi, N. Jones, International Journal of Impact Engineering 117, 113122 (2018)

7. Fehmi Mullaoglu, Fatih Usta, Halit S. Turkmen, Zafer Kazancı, Demet Balkan, Erdem Akay, Procedia Engineering 167, 143 - 150 (2016)

8. A.S. Chepurnenko, V.I. Andreev, B.M. Yazyev, A.N. Beskopylny, MATEC Web of Conferences 67, 06059 (2016)

9. A.N. Beskopylny, E.E. Kadomtseva, G.P. Strelnikov, Y.A. Berdnik, IOP Conf. Ser.: Earth Environ. Sci. 90, 012064 (2017)

10. A.N. Beskopylny, E.E. Kadomtseva, G.P. Strelnikov, IOP Conf. Ser.: Earth Environ. Sci. 90, 012017 (2017)

11. T. Rogovenko, M. Zaitseva, MATEC Web of Conferences 129, 05014 (2017)

12. T. Rogovenko, M. Zaitseva, MATEC Web of Conferences 106, 08011 (2017)

13. D.M. Belen'kii, A.N. Beskopyl'nyi, N.L. Vernezi, L.G. Shamraev, Welding International 11, 642-645 (1997)

14. A. Beskopylny, N. Onishkov, V. Korotkin, Advances in Intelligent Systems and Computing 692, 184-191 (2018) 